Supplement of Geosci. Model Dev., 14, 2205-2220, 2021

https://doi.org/10.5194/gmd-14-2205-2021-supplement

(C) Author(s) 2021. CC BY 4.0 License.

(c) (i)

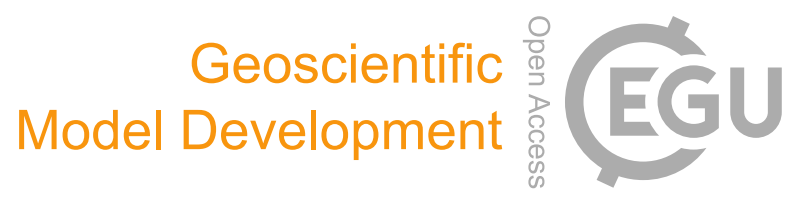

Supplement of

\title{
A new Lagrangian in-time particle simulation module (Itpas v1) for atmospheric particle dispersion
}

Matthias Faust et al.

Correspondence to: Matthias Faust (faust@tropos.de)

The copyright of individual parts of the supplement might differ from the article licence. 


\section{S1 Additional Figures}
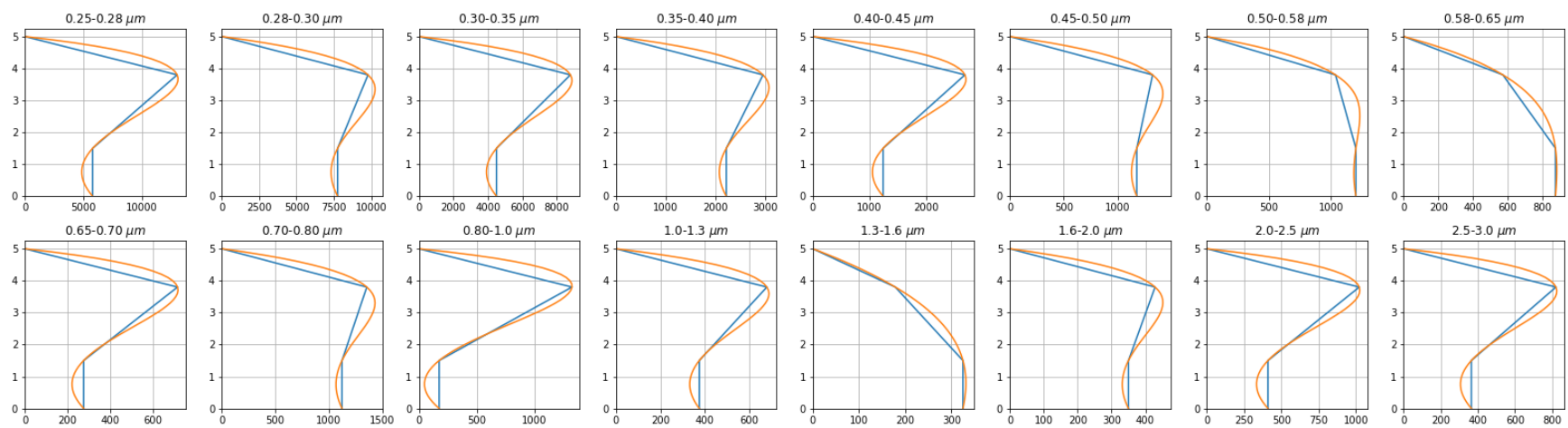

1.0-1.3 $\mu \mathrm{m}$

1.3-1.6 $\mu \mathrm{m}$
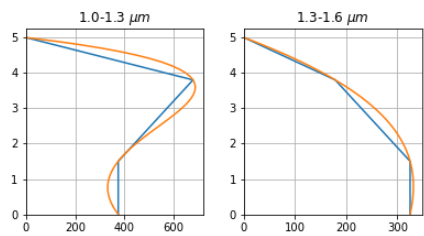

1.6-2.0 $\mu \mathrm{r}$

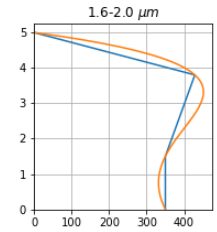

2.0-2.5 $\mu \mathrm{m}$

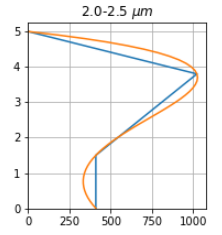

2.5-3.0 $\mu \mathrm{m}$
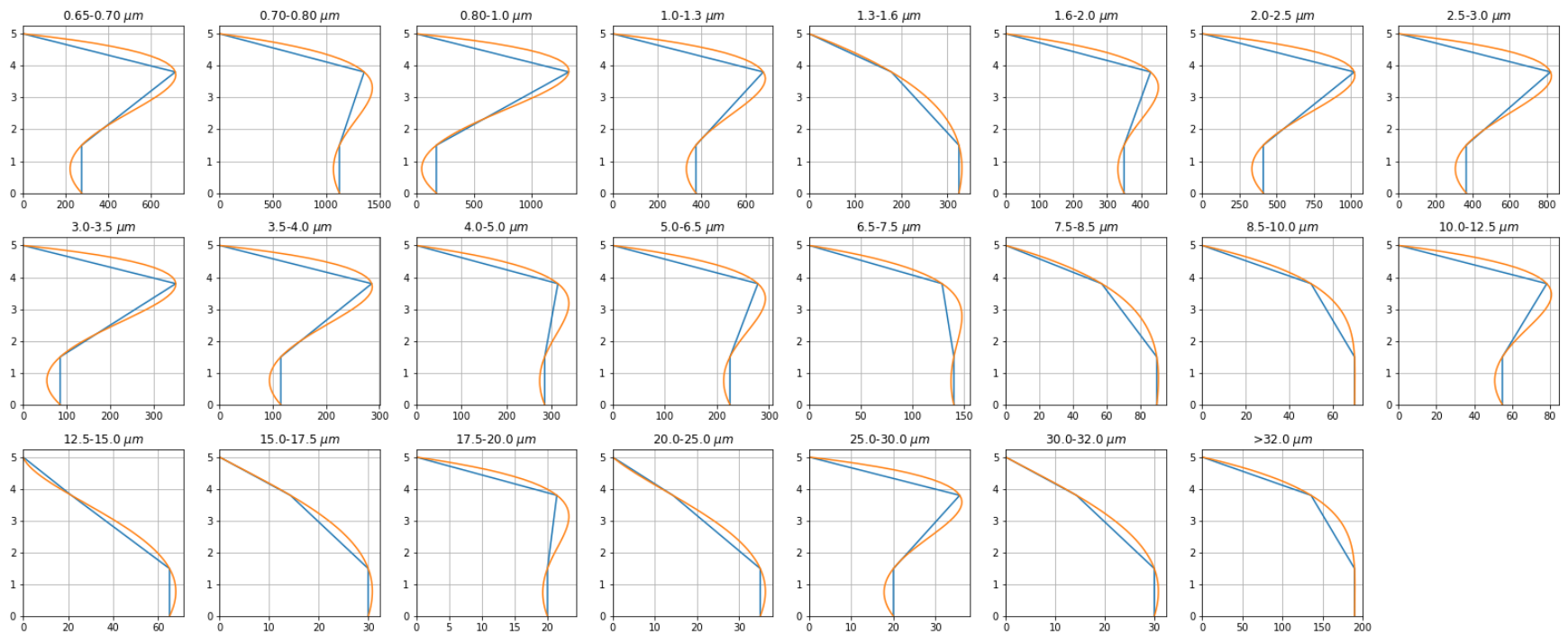

Figure S1. EXP1: Vertical profiles of the particle concentration [particle per litre] for each size bin of the Environmental Dust Monitors; Blue: Measurement; Orange: Polynomial fit; Please note that the scaling of the abscissas vary 


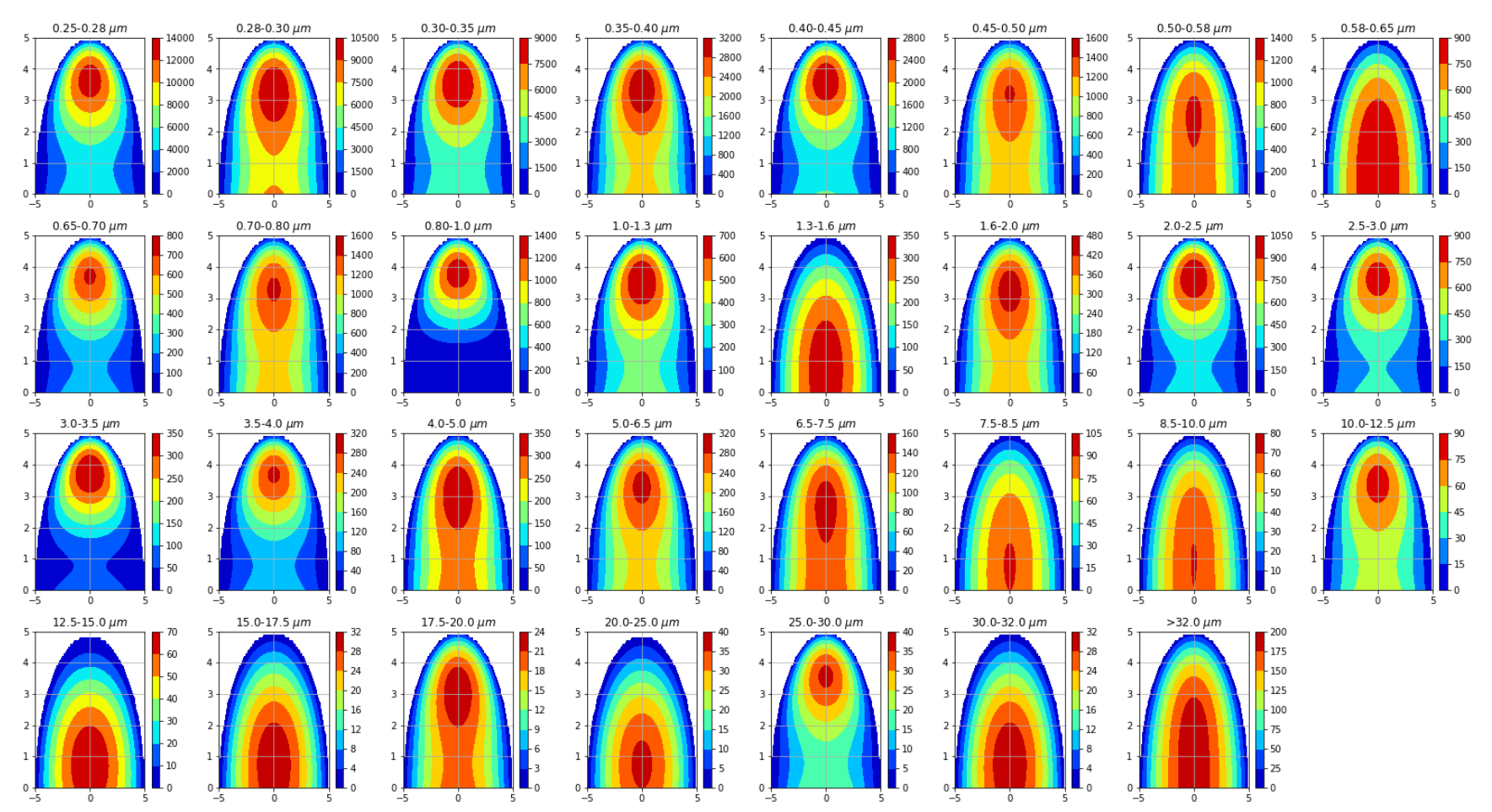

Figure S2. EXP1: Cross-section through the initial particle concentration field [particle per litre] for each size bin of the Environmental Dust Monitors; Please note that the scaling of the colour bar vary 

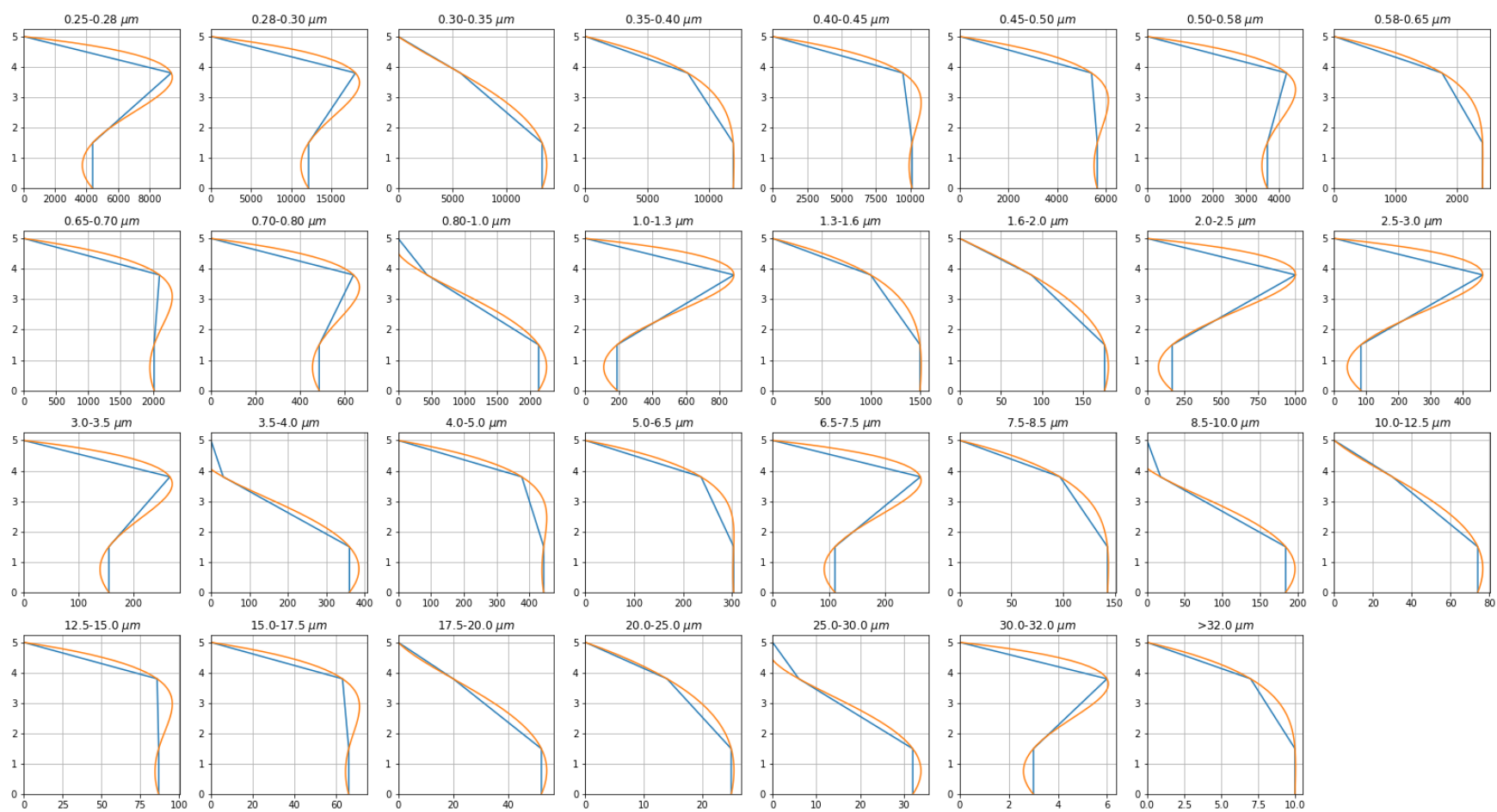

Figure S3. EXP2: Vertical profiles of the particle concentration [particle per litre] for each size bin of the Environmental Dust Monitors; Blue: Measurement; Orange: Polynomial fit; Please note that the scaling of the abscissas vary 

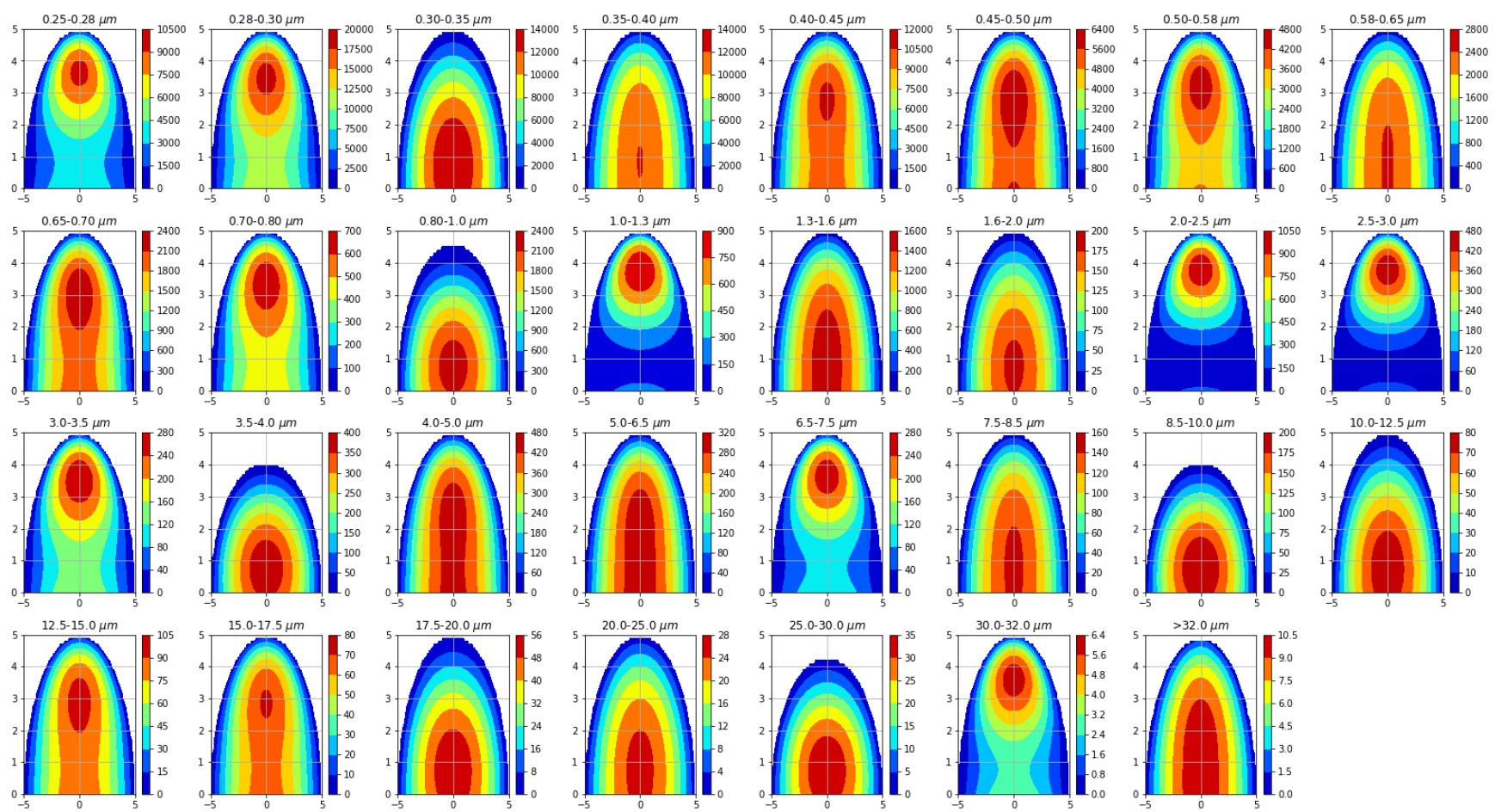

Figure S4. EXP2: Cross-section through the initial particle concentration field [particle per litre] for each size bin of the Environmental Dust Monitors; Please note that the scaling of the colour bar vary 


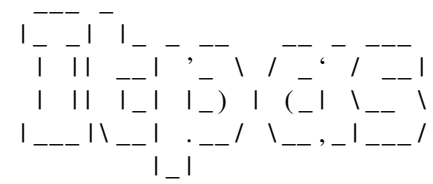

In-time particle simulation (Itpas) module in the framework of the COSMO-Model

The in-time wind information and the prognostic turbulent kinetic energy from the COSMO-model are used to calculate the motion of thousands of aerosol particles inside the turbulent atmospheric boundary layer.

Itpas is written as an extension to the weather forecast model COSMO from the German weather service (DWD), so this software container contains beside of Itpas the complete source code of COSMO (V5.4e).

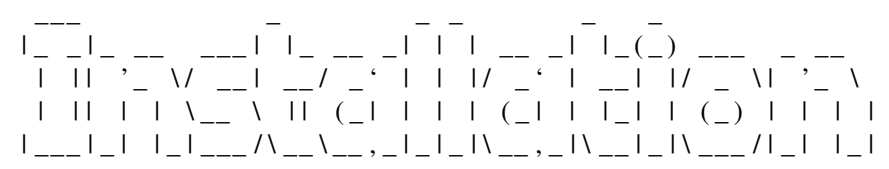

Itpas is integrated into the COSMO-model, so the installation follows the same steps as the standard COSMO installation.

1) set the paths in the Fopts file.

You need an Installation of:

- The DWD-libgrib (availabe for COSMO users at DWD)

- grib_api or eccodes

- NetCDF

2) make (or gmake - j 8 for parallel compiling)

3) The installation worked when the binary cosmo_itpas appears .

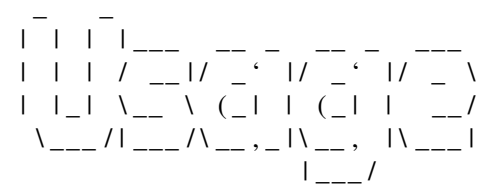

The NAMELIST "partctl"

\begin{tabular}{|c|c|c|c|}
\hline \&PARTCTL & & & ( default) \\
\hline nmax_part & $=500000$, & ! maximum number of particle in the simulation & $(-)$ \\
\hline iseed & $=2711$ & ! seed for the random numbers & $(2711)$ \\
\hline heightover & $=$ 'surface' & ! height in startfile is given definde above'surface, or 'sea' & $($ 'sea') \\
\hline startfile_part & $=, . / \$$ PFILE', & ! path/of/start/file & $(-)$ \\
\hline ydir_part & $=' \$\{$ OUTPUT $\} /,$ & ! path/for/the/output & $(-)$ \\
\hline lpre_output & $=$ true., & ! if true wirte the start position in the netcdf file & $(. \mathrm{false})$. \\
\hline
\end{tabular}




$\begin{array}{lll}\text { lsettling } & \text {.true. } & ! \text { activate particle sedimentation } \\ \text { vd_constant } & =0.0 & ! \text { constant value for the dry deposition velocity. }\end{array}$

The namelist has to be stored in the file INPUT_PART

The startfile

A startfile containing the start positions of the trajectories has to be provided.

It is specified by the user via the namelist parameter startfile part.

It is a text file and its format is strictly defined.

It contains: 3 header lines (no space at the beginning of the lines) and several content lines

\begin{tabular}{llllllll}
\multirow{2}{*}{ time } & of the & \multicolumn{2}{l}{ startfile } & & & \\
\cline { 4 - 7 } & lon & lat & height & npart & diam & dens & emission \\
\hline 1.00 & 1.0 & 2.5 & 5.0 & 1200 & $7.5 \mathrm{e}-7$ & 2650.0 & 1.0 \\
5.00 & 1.0 & 2.5 & 5.0 & 200 & $7.5 \mathrm{e}-7$ & 2650.0 & 0.0
\end{tabular}

- The time is given in hours after model start

- The longitudes are rotated longitudes and can go from -180 to 180 degrees.

They must be expressed in the same referential (pollon, pollat) than the COSMO grid. They can have max. 3 decimal positions.

- The latitudes are rotated latitudes and can go from -90 to 90 degrees.

They must be expressed in the same referential (pollon, pollat) than the COSMO grid.

They can have max. 3 decimal positions.

- The height is above see level or above surface [m], dependig on the namelist setting "heightover"

- npart is the number of particles that were emitted at this time and place.

- diam is the diameter of the particle [m], if gas 0.0

- dens is the density of the particle $[\mathrm{kg} / \mathrm{m} 3]$

- emission is the time in hours that is takes to emit the npart particles note: npart/(emission *3600/dt) need to be an Natural number

COSMO setting s

To run the particle model several settings in different COSMO namelists are necessary.

1) INPUT_ORG

\&RUNCTL

lpart=.TRUE. ! main switch to active the particle model

2) INPUT_PHY

\section{\&PHYCTL}

lforest=. True., ! necessary for the calculation of the dry deposition velocity.

ltur=.TRUE., ! we need the turbulence for the turbulent particle transport

itype_turb=3, ! TKE based turbulence scheme

$13 \mathrm{dturb}=$ true., ! for best results use the $3 \mathrm{~d}$ turbulence scheme

3) INPUT_PART as described above

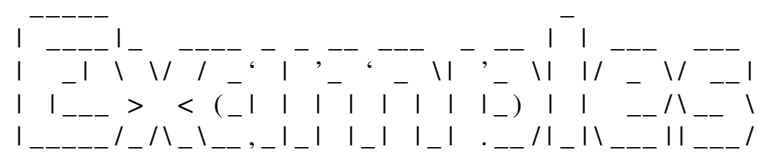


1) A simple trace - what pathway do the air mass take

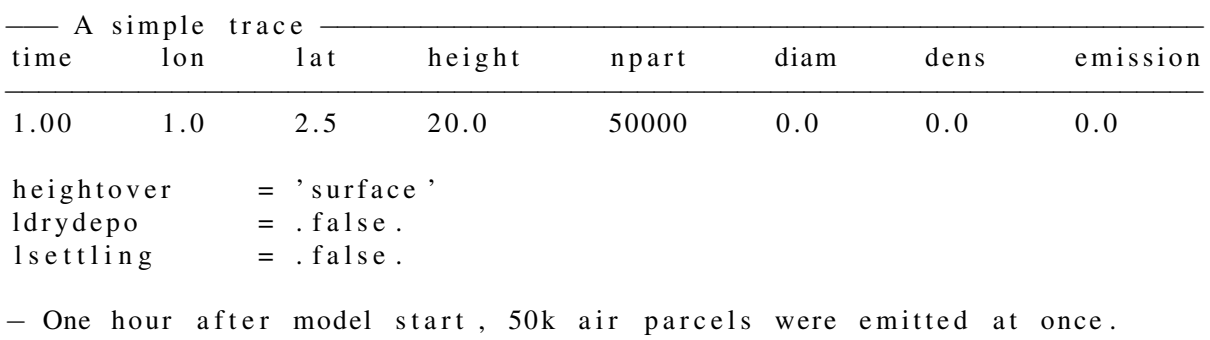

2) emitting point source - a chimney

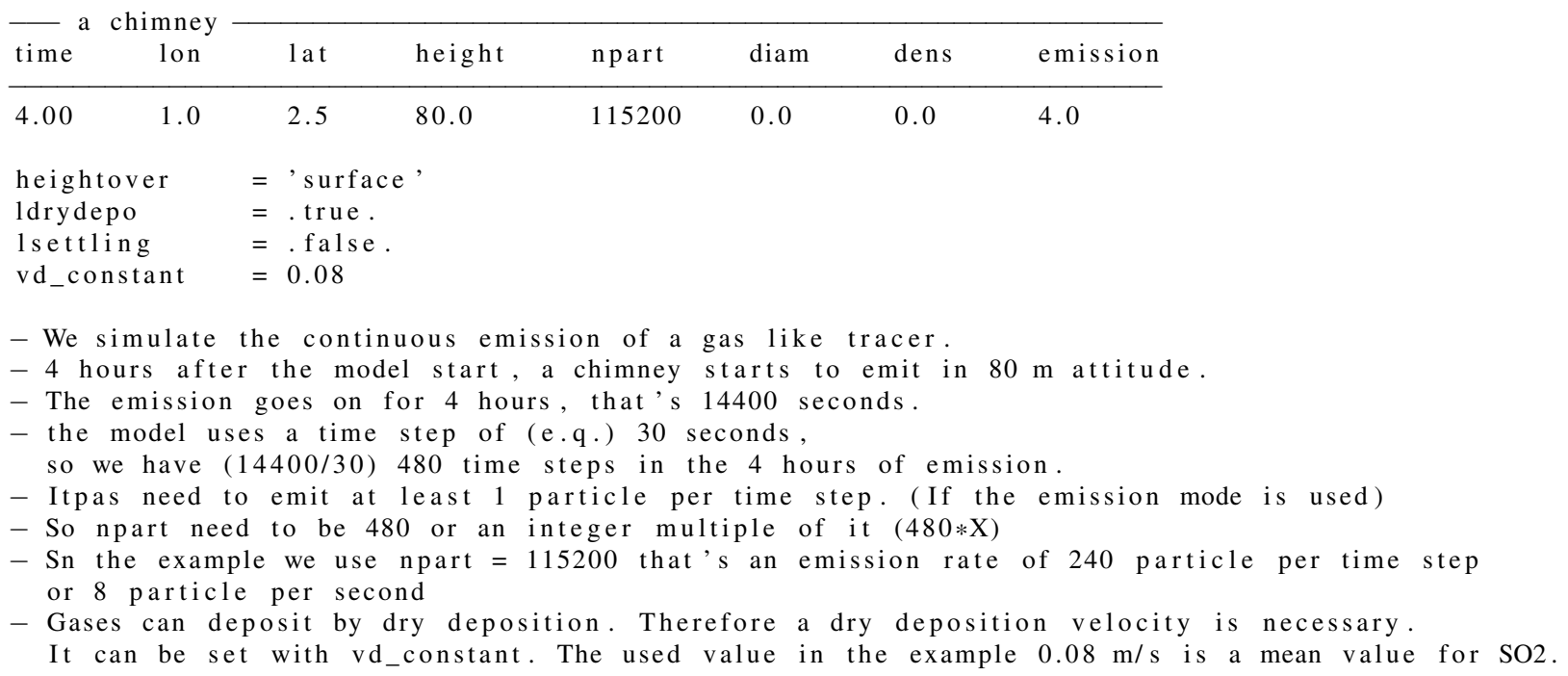

3) a dust plume

\begin{tabular}{|c|c|c|c|c|c|c|c|}
\hline time & lon & lat & height & $\mathrm{npart}$ & diam & dens & emission \\
\hline 2.00 & 1.0 & 2.5 & 5.0 & 1 & $7.5 \mathrm{e}-7$ & 2650.0 & 0.0 \\
\hline
\end{tabular}

$\begin{array}{ll}\text { heightover } & =\text { ' surface, } \\ \text { ldrydepo } & =\text { true. }\end{array}$

lsettling $\quad=$.true .

- for a dust plume, sedimentation is important. Therefore need the particle in the start file a diameter and a density. And lsettling need to be true.

- If you want to include a particle size distribution, write a small script that defines particle sizes and start position based on your needs.

- An example of how this could be realist can you found here: https://github.com/mttfst/dust-bubble 